\title{
Low Complexity BCH Codes with Optimized Interleavers for DQPSK Systems with Laser Phase Noise
}

\author{
Miu Yoong Leong • Knud J. Larsen • Gunnar Jacobsen • Darko Zibar • \\ Sergey Sergeyev • Sergei Popov
}

Received: date / Accepted: date

\begin{abstract}
The presence of high phase noise in addition to additive white Gaussian noise in coherent optical systems affects the performance of forward error correction (FEC) schemes. In this paper, we propose a simple scheme for such systems, using block interleavers and binary Bose-Chaudhuri-Hocquenghem (BCH) codes. The block interleavers are specifically optimized for differential quadrature phase shift keying modulation. We propose a method for selecting $\mathrm{BCH}$ codes that, together with the interleavers, achieve a target post-FEC bit error rate (BER). This combination of interleavers and $\mathrm{BCH}$ codes has very low implementation complexity. In addition, our approach is straightforward, requiring only short pre-FEC simulations to parameterize a model, based on which we select codes analytically. We aim to correct a pre-FEC BER of around $10^{-3}$. We evaluate the accuracy of our approach using numerical simulations. For a target postFEC BER of $10^{-6}$, codes selected using our method
\end{abstract}

This work was supported in part by EU project GRIFFON under Grant 324391, and in part by Vetenskapsrådet under Grant 0379801.

M. Y. Leong and G. Jacobsen

Acreo Swedish ICT, 16425 Stockholm, Sweden and KTH

Royal Institute of Technology, 16440 Stockholm, Sweden

E-mail: miuyoong.leong@acreo.se, gunnar.jacobsen@acreo.se

K. J. Larsen and D. Zibar

Technical University of Denmark (DTU), 2800 Kgs. Lyngby,

Denmark

E-mail: knjl@fotonik.dtu.dk, dazi@fotonik.dtu.dk

S. Sergeyev

Aston University, Birmingham B4 7ET, United Kingdom

E-mail: s.sergeyev@aston.ac.uk

S. Popov

KTH Royal Institute of Technology, 16440 Stockholm, Sweden

E-mail: sergeip@kth.se result in BERs around $3 \times$ target, and achieve the target with around $0.2 \mathrm{~dB}$ extra signal-to-noise ratio.

Keywords Optical fiber communications · Error correction codes · Block codes · Phase noise - Cycle slips

\section{Introduction}

Coherent optical systems have relatively high phase noise $(\mathrm{PN})$ from transmitter and local oscillator (LO) lasers [1-3]. The phase estimation algorithms used in such systems have non-zero probability of cycle slips $[4,5]$. However, these effects are often neglected in coding theory, which focuses on additive white Gaussian noise (AWGN) channels where bit errors are independent identically distributed (i.i.d.) [6-8]. Recently, several FEC approaches have been proposed for systems with PN. In [9-11], the authors consider low-density parity-check (LDPC) codes. In [12], we propose a method for selecting binary $\mathrm{BCH}$ codes using a correlated bivariate binomial model. However, due to the correlation, the selected codes have high overhead, which reduces system throughput. In [13], we use interleaving to decorrelate errors, thereby reducing code overhead. The analysis in [13] is for theoretical uniform interleavers, so the results there are general. However, those interleavers are too complex for practical implementation.

In this paper, we consider a low-complexity practical interleaver that is specifically optimized for differential quadrature phase shift keying (DQPSK) systems. As in [13], interleaving enables us to use codes with lower overheads. However, compared to [13], the interleavers and codes in this paper have much simpler implementations. First, we propose an optimized interleaver and method for selecting binary BCH codes 
for use with this interleaver. We briefly describe the practical implementation of both interleaver and code. Next, we evaluate the accuracy of our method using numerical simulations. Ours is a straightforward method, based on a simple model, that enables us to design lowcomplexity interleavers and binary $\mathrm{BCH}$ codes for any post-FEC BER with little simulation effort. Compared to our method, the approaches in [9-11] achieve better performance by using soft information. However, those schemes are more complex to implement, and require extensive simulations for low post-FEC BERs.

This paper is organized as follows: in Sec. 2, we describe the system model, optimized interleaver, method for code selection, and practical implementation of the interleaved code. Simulation results and discussion are presented in Sec. 3. Finally, the conclusion is in Sec. 4.

\section{Interleavers and Codes}

We consider the system in Fig. 1, which is similar to that in [13] but with a different interleaver/deinterleaver. The DQPSK signal is Gray coded. After differential decoding, an AWGN error of $\pm 90^{\circ}$ gives bit error patterns $\{0101,1010,0110,1001\}$ with probability $1 / 4$ each. A cycle slip of $\pm 90^{\circ}$ gives one bit error. We neglect $180^{\circ}$ errors as these have very low probabilities. The block interleaver/deinterleaver is shown conceptually in Fig. 2. Although they can be implemented as shown in the figure, a much simpler implementation is used in practice, as we describe at the end of this section. In Fig. 2 , data bits are read in by columns, and codes are applied by rows. Identical codes are applied to each interleaver/deinterleaver row. The interleaving degree $\lambda=4$ is chosen to fit the AWGN error patterns, so each AWGN error gives at most one bit error in a deinterleaver row. The same is true for cycle slip errors. The mapping of AWGN and cycle slip errors to deinterleaver rows is shown in Fig. 3. Unlike [13], where random interleaver permutations in combination with specific error realizations may result in a worse distribution of errors after deinterleaving than before, our deterministic mapping always improves the distribution of errors for coding purposes.

We identify suitable codes for this system in three steps. Step 1: we determine a worst-case pre-FEC operating point. Step 2: we record the error statistics for that point. Step 3: we use those statistics to parameterize a model, based on which we select codes analytically.

For Step 1, we proceed in the same way as in $[12,13]$. Namely, for a given laser linewidth-symbol time product, we optimize the length of the Viterbi-Viterbi moving average filter using pre-FEC simulations. Then, for the linewidth variations that we want to accommodate,

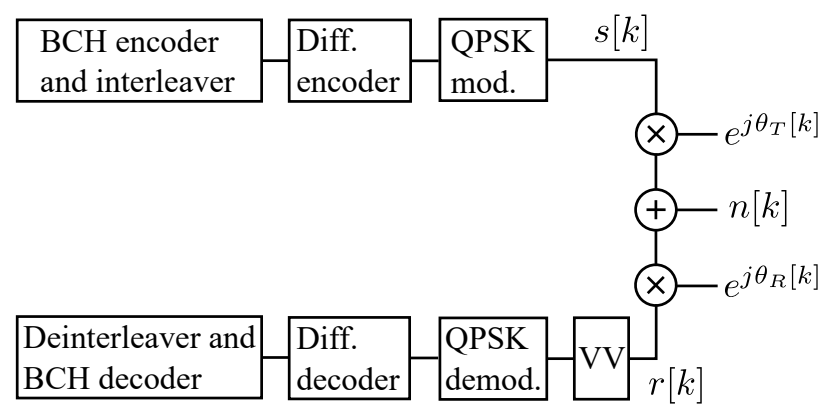

Fig. 1 System model. A random bit sequence is interleaved and $\mathrm{BCH}$ encoded (Fig. 2). It is then differentially encoded and QPSK modulated. This yields signal $s[k]$. Channel impairments are transmitter laser $\mathrm{PN} \theta_{T}[k]$, AWGN $n[k]$, and LO laser $\mathrm{PN} \theta_{R}[k]$. Phase estimation on the received signal $r[k]$ is by Viterbi-Viterbi (VV). The signal is QPSK demodulated and differentially decoded. Finally, it is deinterleaved and $\mathrm{BCH}$ decoded (Fig. 2).

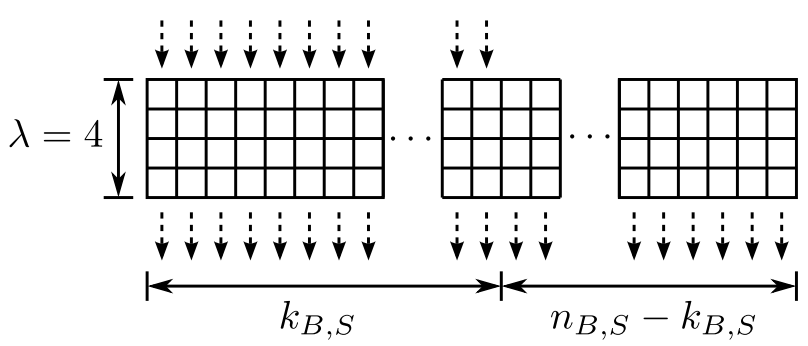

Fig. 2 Conceptual BCH encoder and interleaver. First, $\lambda$. $k_{B, S}$ data bits are read into the interleaver columnwise. Then $\mathrm{BCH}$ encoding is done on each row. Finally, the $\lambda \cdot n_{B, S}$ coded bits are read out columnwise. The interleaving degree $\lambda$ (number of rows) is fixed to 4 . The $\mathrm{BCH}$ decoder and deinterleaver are conceptually similar. In these, $\lambda \cdot n_{B, S}$ bits are read in columnwise, decoded in rows, and the resulting $\lambda \cdot k_{B, S}$ bits are read out columnwise.

\begin{tabular}{|c|c|c|c|c|c|c|c|c|c|c|c|}
\hline A1 & A2 & A3 & A4 & A5 & A6 & A7 & A8 & C1 & C2 & C3 & C4 \\
\hline 0 & 1 & 0 & 1 & X 0 & X 1 & X 1 & X 0 & 1 & 0 & X 0 & X 0 \\
\hline 1 & 0 & 1 & 0 & X 1 & X 0 & X 0 & X 1 & 0 & 1 & X 0 & X 0 \\
\hline 0 & 1 & 1 & 0 & 0 X & $1 \mathrm{X}$ & $0 \mathrm{X}$ & $1 \mathrm{X}$ & 0 & 0 & $1 \mathrm{X}$ & $0 \mathrm{X}$ \\
\hline 1 & 0 & 0 & 1 & $1 \mathrm{X}$ & $0 \mathrm{X}$ & $1 \mathrm{X}$ & $0 \mathrm{X}$ & 0 & 0 & $0 \mathrm{X}$ & $1 \mathrm{X}$ \\
\hline
\end{tabular}

Fig. 3 Bit error patterns in the deinterleaver before $\mathrm{BCH}$ decoding. Rows correspond to the rows of the deinterleaver in Fig. 2. A " 1 " indicates the presence of a bit error, "0" means no bit error, and "X" means don't care. (A1-A4) are AWGN error patterns that start on odd (1st, 3rd, 5th, ...) DQPSK symbols, (A5-A8) are AWGN patterns that start on even (2nd, 4th, 6th, ...) symbols, and (C1-C4) are cycle slip patterns.

we simulate a worst-case "poor phase estimate (PE)" curve (Fig. 4).

The rest of this section describes Steps 2 and 3 . The code $\operatorname{BCH}\left(n_{B, S}, k_{B, S}\right)$ is applied to each interleaver/deinterleaver row. It has block length $n_{B, S}$ bits, of which $k_{B, S}$ are data bits. It corrects up to at least $\tau$ bit errors and may be a shortened code $[8,12]$. 


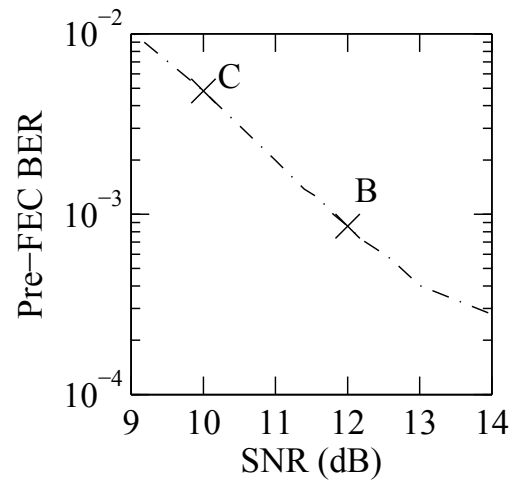

Fig. 4 Poor phase estimate (PE) pre-FEC BER with total linewidth $\Delta \nu_{N}=19.6 \mathrm{MHz}$. We select codes for "poor PE pre-FEC" points C (signal-to-noise ratio (SNR) $10 \mathrm{~dB}$ ) and B (SNR $12 \mathrm{~dB}$ ). The SNR is for symbols $r[k]$ in Fig. 1 .

For Step 2, we record the following error statistics: 1. probability of an AWGN bit error in a deinterleaver row $p_{G}, 2$. probability of a cycle slip bit error in a deinterleaver row $p_{C}$, and 3 . correlation coefficient $\rho$. We use the same symbols $p_{G}, p_{C}$, and $\rho$ as in [12], but redefine the quantities they represent. We note that each of the AWGN error patterns in Fig. 3(A1-A8) is equiprobable. Similarly, each of the cycle slip patterns in Fig. $3(\mathrm{C} 1-\mathrm{C} 4)$ is equiprobable. Thus we see from Fig. 3 that bit errors are evenly distributed between deinterleaver rows. Therefore, using identical codes on each row is optimal in the sense that no overhead is wasted on rows with few errors. This property leads to the very simple implementation that we briefly describe at the end of this section.

We now define the error statistics for each row of Fig. 3. For the $d$-th deinterleaver frame of $4 n_{B, S}$ bits, we record the probability of an AWGN bit error on row 1 of the deinterleaver as

$p_{G, 1}[d] \triangleq \frac{\text { number of occurrences of A2, A4, A6, A7 }}{4 n_{B, S}}$.

Similarly, the probability of a cycle slip bit error on row 1 of the deinterleaver is

$p_{C, 1}[d] \triangleq \frac{\text { number of occurrences of } \mathrm{C} 1}{4 n_{B, S}}$.

Let $p_{G, 1}$ be the mean of $p_{G, 1}[d]$ over the $D$ interleaver frames used in pre-FEC simulations, i.e.

$p_{G, 1}=\frac{1}{D} \sum_{d=1}^{D} p_{G, 1}[d]$.

Similarly,

$p_{C, 1}=\frac{1}{D} \sum_{d=1}^{D} p_{C, 1}[d]$.
Due to the influence of AWGN on Viterbi-Viterbi phase estimation, $p_{G, 1}$ and $p_{C, 1}$ are correlated [12]. We denote their sample correlation coefficient as $\rho_{1}$. Doing this for all rows in Fig. 3 yields $p_{G, r}, p_{C, r}$, and $\rho_{r}$, where $r \in\{1,2,3,4\}$.

Due to the symmetry of Fig. 3 , in the limit, $p_{G, 1}=$ $p_{G, 2}=p_{G, 3}=p_{G, 4} \triangleq p_{G}$. Likewise, $p_{C, 1}=p_{C, 2}=$ $p_{C, 3}=p_{C, 4} \triangleq p_{C}$, and $\rho_{1}=\rho_{2}=\rho_{3}=\rho_{4} \triangleq \rho$. To obtain an accurate estimate of $p_{G}, p_{C}$, and $\rho$, we therefore use all rows and interleaver frames as follows:

$p_{G}=\frac{1}{4 D} \sum_{r=1}^{4} \sum_{d=1}^{D} p_{G, r}[d]$

$p_{C}=\frac{1}{4 D} \sum_{r=1}^{4} \sum_{d=1}^{D} p_{C, r}[d]$

and

$\rho=\frac{\sum_{r, d}\left(p_{G, r}[d]-p_{G}\right)\left(p_{C, r}[d]-p_{C}\right)}{\sqrt{\sum_{r, d}\left(p_{G, r}[d]-p_{G}\right)^{2} \sum_{r, d}\left(p_{C, r}[d]-p_{C}\right)^{2}}}$.

For Step 3, we parameterize the correlated bivariate binomial probability density function $(\mathrm{PDF}) \operatorname{Pr}\left(Y_{G}=\right.$ $\left.y_{G}, Y_{C}=y_{C}\right)$ in [12] with the redefined parameters $p_{G}$, $p_{C}$, and $\rho$ in (5)-(7). Additionally, in the equation for $\operatorname{Pr}\left(Y_{G}=y_{G}, Y_{C}=y_{C}\right)[12]$, we replace all instances of $n_{B, S}$ by $4 n_{B, S}$.

The decoding algorithm is assumed to be of the bounded-distance type correcting up to $\tau$ errors and leaving the received sequence unchanged in the case of more than $\tau$ errors. In other words, neglecting the possibility of decoding to a wrong codeword [12], a code block (deinterleaver row) which has $e$ bit errors before $\mathrm{BCH}$ decoding will have no errors after $\mathrm{BCH}$ decoding if $e \leq \tau$. If $e>\tau$ there will be, on average, $e\left(k_{B, S} / n_{B, S}\right)$ bit errors in the $k_{B, S}$ bits after decoding.

The total number of bit errors in a deinterleaver row is $y_{G}+y_{C}$. Thus, the probability of a non-decodable codeword is

$P_{B}=\sum_{\left(y_{G}, y_{C}: y_{G}+y_{C} \geq \tau+1\right)} \operatorname{Pr}\left(Y_{G}=y_{G}, Y_{C}=y_{C}\right)$.

To relate $P_{B}$ to post-FEC BER, we apply the same approximations as in [12]. Assuming $n_{B, S}\left(p_{G}+p_{C}\right) \ll \tau$, we approximate the probability tail by its three largest boundary terms,

$$
P_{B} \approx \sum_{\left(y_{G}, y_{C}: \tau+3 \geq y_{G}+y_{C} \geq \tau+1\right)} \operatorname{Pr}\left(Y_{G}=y_{G}, Y_{C}=y_{C}\right)
$$


Three terms are used because the $\mathrm{PDF}$ of $\operatorname{Pr}\left(Y_{G}=\right.$ $\left.y_{G}, Y_{C}=y_{C}\right)$ is two-dimensional. Post-FEC BER is

$P_{\text {post }} \approx\left(\frac{\tau+1}{n_{B, S}}\right) P_{B}$.

Using (5)-(7) from pre-FEC simulations and (9)(10), we calculate the required $\tau$ to meet a target postFEC BER for a chosen block length $n_{B, S}$. Our method does not restrict the choice of block length. The combination $n_{B, S}$ and $\tau$ specifies the $\mathrm{BCH}$ code.

We now briefly describe a simple implementation of the code and interleaver/deinterleaver. While they may be implemented as shown in Fig. 2, a simpler solution exists. The key to recognizing this is to note that, if the generator polynomial for the $\operatorname{BCH}\left(n_{B, S}, k_{B, S}\right)$ code is $g(X)$, then the generator polynomial for the interleaved code is $g\left(X^{\lambda}\right)$ [8]. Therefore, an encoder and decoder for the interleaved code can be obtained from that of the base code, by replacing each shift register in the base implementation with $\lambda$ shift registers. This causes the circuit to operate on successive rows during successive clock cycles. Thus, the interleaved code can be implemented with the same hardware as the base code plus additional shift registers.

Another popular interleaver is the $S$-random interleaver [14]. Compared to the block interleaver in this section, $S$-random interleavers are more complex to implement. Since the permutation in $S$-random interleavers is not regular, their implementation cannot be reduced to the addition of shift registers. Instead, their permutation must be either pseudo-randomly generated or stored in a lookup table.

The uniform interleaver in [13] is a theoretical construct. Implementation would require an ensemble of pseudo-random generators, which is not practical.

\section{Results and Discussion}

We evaluate our method using Monte-Carlo simulations for the system in Fig. 1. As an example to illustrate the use of our scheme to accommodate significant linewidth variations, we assume that a 41-tap moving average filter for Viterbi-Viterbi was optimized for symbol rate $1 / T_{S}=28$ Gbaud and combined transmitter-and-LO laser linewidths $\Delta \nu_{N}<100 \mathrm{kHz}$. We further assume that the worst-case pre-FEC performance for the system occurs with a linewidth of $\Delta \nu_{N}=19.6 \mathrm{MHz}$, and simulate this numerically as "poor PE pre-FEC" in Fig. 4. Pre-FEC BER and error statistics (described in Sec. 2 ) are calculated using $4 \times 10^{6}$ bits. Simulations are modeled in VPI [15].

In our example, we select codes for points $\mathrm{B}$ and $\mathrm{C}$ in Fig. 4. We aim for a target post-FEC BER of $10^{-6}$
Table 1 Codes for post-FEC simulations in Fig. 5. Codes "B1/C1" are selected using the method in [12], "B2/C2" are selected using [13] for interleaver length $L=4$ code blocks, and "B4/C4" are selected using Sec. 2. Overhead is $\left(n_{B, S}-\right.$ $\left.k_{B, S}\right) / k_{B, S}$.

\begin{tabular}{cccc}
\hline $\begin{array}{c}\text { Post-FEC curve } \\
\text { in Fig. 5 }\end{array}$ & Code & $\tau$ & $\begin{array}{c}\text { Overhead } \\
(\%)\end{array}$ \\
\hline B1 & $\mathrm{BCH}(8190,7891)$ & 23 & 3.8 \\
B2 & $\mathrm{BCH}(8190,7943)$ & 19 & 3.1 \\
B4 & $\mathrm{BCH}(8190,7956)$ & 18 & 2.9 \\
C1 & $\mathrm{BCH}(8190,7228)$ & 75 & 13.3 \\
C2 & $\mathrm{BCH}(8190,7332)$ & 67 & 11.7 \\
C4 & $\mathrm{BCH}(8190,7358)$ & 64 & 11.3 \\
\hline
\end{tabular}

using a block length of $n_{B, S}=8190$ bits. Following the semi-analytical method in Sec. 2, we obtain codes B4 and C4 listed in Table 1. We implement each code in turn into the simulator (Fig. 1). Monte-Carlo simulations thus yield two post-FEC BER curves ("B4" and "C4" in Fig. 5). Close to the target signal-to-noise ratios (SNRs) $(10 \pm 0.4 \mathrm{~dB}$ for "C" and $12 \pm 0.4 \mathrm{~dB}$ for "B"), post-FEC BERs are calculated on $10^{8}$ postFEC bits. At lower SNRs (higher BERs), $10^{7}$ post-FEC bits are used to shorten simulation times. Since $\mathrm{C} 4$ is a stronger code, its post-FEC curve is steeper than B4. The error floor caused by $\mathrm{PN}$ is effectively mitigated by the $\mathrm{BCH}$ codes, which do not themselves have an error floor. The codes selected with our method give BERs around $2 \times$ the post-FEC target. They meet the target post-FEC BER with around $0.1 \mathrm{~dB}$ extra SNR (target $\mathrm{SNR}$ is $10 \mathrm{~dB}$ for $\mathrm{C} 4$ and $12 \mathrm{~dB}$ for $\mathrm{B} 4$ ), which is a negligible difference in practical systems. In other words, our method accurately identifies minimum-overhead $\mathrm{BCH}$ codes that, together with the optimized block interleaver, achieve performance close to target.

In the example above, we use the same symbol rate, linewidth, filter, and block length as in $[12,13]$. At a particular operating point (e.g. "B" in Fig. 5), a shorter block length would generally require a code with higher overhead for the same target post-FEC BER.

At lower post-FEC BERs, the leading-order approximation in (9) becomes more accurate, i.e. approximation error is less at practical post-FEC BERs of $10^{-15}$ than in our example with $10^{-6}$. On the other hand, any inaccuracies in fitting the model $\operatorname{Pr}\left(Y_{G}=y_{G}, Y_{C}=y_{C}\right)$ based on pre-FEC simulations become more apparent at lower post-FEC BERs. As we simulate at most $10^{8}$ post-FEC bits due to simulation limitations, the model has not been verified down to post-FEC BERs of $10^{-15}$. Nevertheless, the accuracy of our results shows that the model captures the main behavior of the system.

For comparison, we select code B2 using [13] with a uniform interleaver of length $L=4$ code blocks. Codes 


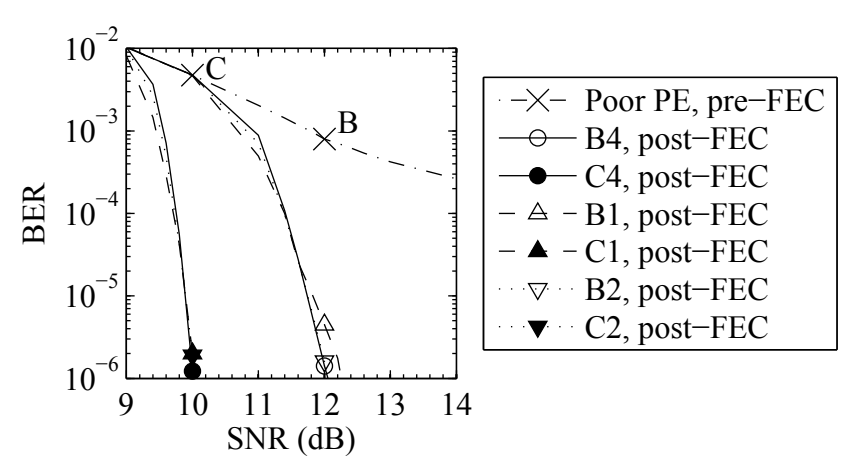

Fig. 5 BER performance with total linewidth $\Delta \nu_{N}=19.6$ MHz. "Poor PE pre-FEC" is the same as Fig. 4. The codes used for post-FEC simulations are listed in Table 1. Codes "B4/C4" are selected using the method in Sec. 2, "B1/C1" are selected using the method in [12], and "B2/C2" are selected using [13] for interleaver length $L=4$ code blocks.

$\mathrm{B} 2$ and B4 therefore have the same interleaver frame length of $4 n_{B, S}$ bits, but the former uses theoretical uniform interleaving [13] whereas the latter uses lowcomplexity practical block interleaving (Sec. 2). Both have similar error correcting capabilities $\tau$ and overheads (Table 1), and similar post-FEC performance (Fig. 5). This is also the case for codes $\mathrm{C} 2$ and $\mathrm{C} 4$ which again have interleaver frame lengths of $4 n_{B, S}$ bits, where the former uses theoretical uniform interleaving [13] and the latter uses low-complexity practical block interleaving (Sec. 2). In other words, our method results in a simple practical implementation with similar code overhead as theoretical uniform interleaving. In addition, we select code B1 using the method in [12] with no interleaving. As expected, both B2 and B4 have lower overheads than B1. In fact, post-FEC simulations for B1 (Fig. 5) suggest that a code with even higher overhead is needed to achieve the BER target of $10^{-6}$ at $12 \mathrm{~dB}$ SNR. Likewise, we also select code C1 using the method in [12] with no interleaving, and find that both $\mathrm{C} 2$ and $\mathrm{C} 4$ have lower overheads than $\mathrm{C} 1$.

At SNRs other than the target points B and C, the post-FEC BERs in Fig. 5 depend on the choice of code, the pre-FEC error statistics (described in Sec. 2, [12, 13]) of the different schemes at those SNRs, and the fit of those statistical models.

Coding and interleaving introduce additional processing time into the system. While exact numbers are implementation-specific, we can obtain an order-of-magnitude estimate as follows. We assume that encoding is done by shifting bits into a linear feedback shift register (LFSR) and simultaneously into the channel [8], so latency is negligible. We assume that decoding is dominated by syndrome computation and error-location numbers computation, where the former is done as bits are shifted into an $n_{B, S}$ bit buffer and simultaneously into

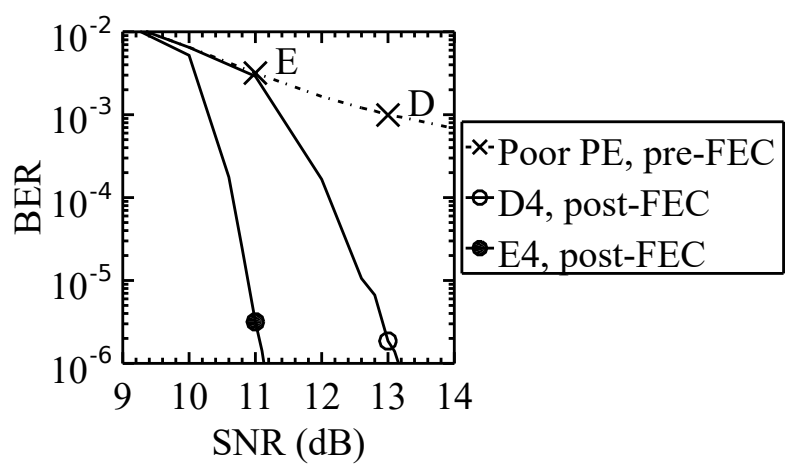

Fig. 6 BER performance with total linewidth $\Delta \nu_{N}=25.2$ MHz. Codes "D4/E4" are selected using the method in Sec. 2. Code D4 is $\operatorname{BCH}(8190,7930)$ with $\tau=20$ and overhead $3.3 \%$. Code $\mathrm{E} 4$ is $\mathrm{BCH}(8190,7579)$ with $\tau=47$ and overhead $8.1 \%$.

LFSRs, and the latter as they are shifted out [8], thus totaling $2 \lambda n_{B, S}$ clock cycles. For a bitrate of $2 / T_{S}=56$ Gbps, $\lambda=4$, and codes B4/C4 in Table 1 , this is approximately $1 \mu \mathrm{s}$. By comparison, it takes approximately $1 \mathrm{~ms}$ for a signal to traverse $200 \mathrm{~km}$ of fiber.

We also evaluate our method for the more extreme linewidth of $\Delta \nu_{N}=25.2 \mathrm{MHz}$ (Fig. 6), which has a pre-FEC BER floor just below the $10^{-3}$ level that our method aims to correct. Using the method in Sec. 2 for a target post-FEC BER of $10^{-6}$, we obtain codes D4 and E4 in Fig. 6. Simulations yield BERs around $3 \times$ target, and achieve the target with around $0.2 \mathrm{~dB}$ extra SNR. This is similar to the results in Fig. 5.

\section{Conclusion}

In this paper, we present a block interleaver that is optimized for DQPSK systems, and a semi-analytical method for selecting binary BCH codes for use with this interleaver. This combination of interleavers and codes has very low implementation complexity. Our approach is straightforward and requires only modest simulation effort to extract statistics from pre-FEC simulations, based on which codes are selected analytically. As an example, we evaluate our approach for a 28 Gbaud system with linewidths ranging from $<100 \mathrm{kHz}$ to 25.2 $\mathrm{MHz}$. For a target post-FEC BER of $10^{-6}$, the codes identified with our method give BERs around $3 \times$ target, and achieve the target with around $0.2 \mathrm{~dB}$ extra SNR.

Future research could consider higher-order modulation formats such as those in [16-18]. This would introduce additional effects and considerations. For example, in general circular differential quadrature amplitude modulation, constellation points are arranged on rings of different amplitudes. Different rings can have 
different numbers of points at arbitrary rotations, and perfect Gray coding is not always possible. This gives more degrees of freedom when optimizing the constellation and more factors to consider when selecting codes. Other interesting effects to consider could include nonlinear phase noise [19], and the interplay between $\mathrm{PN}$ and chromatic dispersion [20,21].

\section{References}

1. I. Garrett, G. Jacobsen, Optoelectronics, IEE Proceedings J 136(3), 159 (1989)

2. G. Jacobsen, I. Garrett, Electron. Lett. 21(7), 268 (1985)

3. F. Mogensen, G. Jacobsen, H. Olesen, Optical and Quantum Electronics 16(2), 183 (1984)

4. T. Pfau, in Proc. OFC (2014). W4K.1

5. M.G. Taylor, J. Lightw. Technol. 27(7), 901 (2009)

6. A. Leven, L. Schmalen, in Proc. ECOC (2013). We.2.C.1

7. F. Chang, K. Onohara, T. Mizuochi, Communications Magazine, IEEE 48(3), S48 (2010)

8. S. Lin, D.J. Costello, Error Control Coding, 2nd edn. (Prentice Hall, 2004)

9. L. Schmalen, J. Lightw. Technol. 33(7), 1319 (2015)

10. T. Koike-Akino, K. Kojima, D. Millar, K. Parsons, Y. Miyata, W. Matsumoto, T. Sugihara, T. Mizuochi, in Proc. OFC (2014). M3A.3

11. F. Yu, N. Stojanovic, F. Hauske, D. Chang, Z. Xiao, G. Bauch, D. Pflueger, C. Xie, Y. Zhao, L. Jin, Y. Li, L. Li, X. Xu, Q. Xiong, in Proc. ECOC (2011). We.10.P1.70

12. M.Y. Leong, K.J. Larsen, G. Jacobsen, S. Popov, D. Zibar, S. Sergeyev, J. Lightw. Technol. 32(21), 4048 (2014)

13. M.Y. Leong, K.J. Larsen, G. Jacobsen, S. Popov, D. Zibar, S. Sergeyev, IEEE Photon. Technol. Lett. $\mathbf{2 7}(7), 685$ (2015)

14. S. Dolinar, D. Divsalar, TDA Progress Report 42-122 (1995)

15. (2015). URL www.vpiphotonics.com

16. W.J. Weber, III, IEEE Trans. Commun. 26(3), 385 (1978)

17. T. Pfau, X. Liu, S. Chandrasekhar, in Optical Communication (ECOC), 2011 37th European Conference and Exhibition on (2011), pp. 1-3

18. R. Krishnan, A. Graell i Amat, T. Eriksson, G. Colavolpe, IEEE Trans. Commun. 61(12), 5056 (2013)

19. J.P. Gordon, L.F. Mollenauer, Opt. Lett. 15(23), 1351 (1990)

20. T. Xu, G. Jacobsen, S. Popov, J. Li, A.T. Friberg, Y. Zhang, Opt. Express 19(8), 7756 (2011)

21. G. Jacobsen, T. Xu, S. Popov, J. Li, A.T. Friberg, Y. Zhang, Opt. Express 19(15), 14487 (2011) 